\title{
Study on the Application of Transmission Technology in Information and Communication Engineering
}

\author{
Xinhong Yang ${ }^{1, \text { a }}$ \\ ${ }^{1}$ Shenyang Polytechnic College, Shenyang, Liaoning, 110045 \\ ${ }^{a}$ email
}

Keywords: Transmission Technology; Information and Communication Engineering; Application

\begin{abstract}
At present, with the development of social economy and science and technology, the application of computer network technology in social life is more and more extensive. As an important part of network communication technology, transmission technology has become the main research direction and people are constantly exploring and optimizing. In the communication engineering industry, the application of advanced transmission technology has been gradually broadened, such as wired optical information transmission technology and wireless information transmission technology, etc., all occupy the main position in Chinese communication engineering industry. This paper first introduces the current development status of information and communication engineering and then the further introduction of the current main transmission technology is discussed based on this, including light transmission technology and wireless information transmission technology. Then this paper further introduced the transmission technology in the communications engineering industry applications. Finally, it explores the trend and prospect of transmission technology in information and communication engineering.
\end{abstract}

\section{Introduction}

In today's era, information and communication engineering has received more and more attention, and the application of information transmission technology in the social life more and more widely, is constantly improving and innovating. In this context, more and more advanced information transmission technology is being developed and applied, become the Internet application after another major hot field of information technology. As a result, the new information transmission technology has also been placed more expectations, it needs more in-depth and extensive research, so as to continuously promote the development of newer and more practical technology, promote the information and communication engineering industry to a new level [1]. In the information and communication engineering industry, the most important technical obstacle is the non-destructive transmission of information, with the further development of optical fiber communication and information transmission technology has a new direction of development. Therefore, the follow-up development of information and communication engineering industry and the current development of information transmission technology has a very close relationship. This paper focuses on the application of optical fiber transmission technology, and also briefly describes the development of wireless communication technology, in order to information transmission technology in communication engineering applications in-depth study.

\section{Information and Communication Engineering Overview}

Information and communication engineering (ICT) is a kind of industry with broad knowledge and wide range of applications, including wireless communication and wired communication. It mainly relies on a variety of engineering methods to achieve information transmission, and strive to achieve the information transfer process Non-destructive, fast goals, so that people more easily realize the network of life, intelligent requirements [2]. Communication engineering across the modern sound, light, electricity and many other industries, and is closely related with the network computer industry to hardware facilities for the material basis for the transfer of modern information technology as the goal, which uses the relevant technical software for information 
processing and Transmission, and strengthen the daily communication of people's ability to communicate. At present mainly rely on optical fiber transmission technology and wireless transmission technology to protect the two major categories of communication information transmission of the validity and integrity. Optical fiber transmission technology to build, making information through the high reliability of broadband transmission, the formation of information superhighway, and wireless transmission technology is more flexible, able to get rid of the rigidity of cable transmission, is also gaining favor.

\section{Fiber Optic Information Transmission Technology}

Optical fiber transmission technology overview. Optical fiber transmission technology refers to the use of light as a medium means of data transmission of a wired information transmission technology. Optical fiber transmission technology operating frequency is very high, compared to the traditional copper transmission frequency 1.54MHZ / S, with 2.5GB / S transmission frequency is undoubtedly more efficient, which is also known as optical fiber transmission technology "information highway" The main reason [3]. Optical fiber transmission technology has the advantage not only in the above-mentioned high transmission rate, and the optical fiber also has a large information storage capacity, and corresponding to the low cost of fiber, low consumption, on the whole, is the most development potential of information transfer method. Since Chinese accession to the WTO, with the rapid spread of Internet technology in the global spread of rapid, triple play and 4G era also makes the development of information transmission more quickly. In this context, the optical fiber transmission technology in the information and communication engineering has been more widely used, as modern communication engineering in one of the most important way, with the global wave of Internet development and modern optical fiber communication technology also supports optical transmission Technology towards a more complete, more stable direction [4].

The main principle of optical fiber communication technology is the use of optical fiber, the use of the signal transmitter will need to transfer out of the information into electrical signals, and then the signal modulated laser emitters to the laser beam, according to the frequency of electrical signal to adjust the laser intensity, and then By the laser beam emitted. After the information is transmitted, the laser beam received by the information receiver is converted into an electrical signal by the detector after the laser beam is received by the signal receiver, and then the original information is restored by mediation. This process consists of five major processes, namely, transmit signals, and wave signals, transmission signals, separate signals and receive signals. The transmission capacity of optical fiber transmission technology is much higher than the traditional technology, first because the frequency is higher than the original frequency, and in the transmission process due to fiber loss is lower than the cable. Second, the optical fiber itself is an electrical insulator, in the course of the need to pay attention to the ground loop, not prone to skewers. Thirdly, the optical fiber transmission technology has higher security, because in the optical fiber transmission process is in the fiber, so only leaks the signal will not have the information leakage question.

The main applications of optical fiber transmission on the communications engineering. At present, the optical fiber transmission technology is in the ultra-long distance transmission, because MSC have a long distance between, leading to the development of fiber in the long-distance transmission unsatisfactory. Therefore, without increasing the number of communication cables under the premise of only the need to establish some new wavelength optical channel, will be able to achieve long-distance transmission targets. This new method not only realized the great increase of information transmission system capacity, but also can save considerable cost, which is widely used in information and communication engineering construction [5].

For short distance optical fiber transmission, at present, in the backbone of the backbone transmission network, the more important nodes are generally distributed in the city center, and the main means of installation is achieved through the pipeline. Fiber resources are limited, how to transmit and use has become more concerned about the issue. Once again, the WDM system has attracted more and more people. If in the transmission process needs to take the SDH and the ASON 
two kinds of unifies the way, then the ASON system may in the SDH transmission network and under the G.782 standard optical transmission network realizes. To achieve integration with the existing network system, but also the need for further development.

fiber optic transmission technology application of the main features. Now with the continuous development of science and technology, the market application of the transmission of information products is showing the characteristics of its volume shape are constantly smaller. For example, people now indispensable mobile phone or light receiver, the volume is constantly smaller. This can not only save costs, but also to facilitate the use of people, but also conducive to the latter part of the project for the maintenance and repair of the network [6].

Functional diversification refers to the transmission technology products have been integrated with a variety of business characteristics, the original can be a variety of independent equipment to work together into a device. As a result, it can greatly enhance the utilization of transmission equipment, saving resources, but also just in line with the current market trend of continuous development and improve the product cost.

In the traditional, communication equipment is mainly used for information transmission, and you want to give it more functions must strengthen the product integration, which not only help to improve the added value of products, but also can enhance the economic benefits of communication equipment. And this requires the communication device not only for information transfer, but also has the function of other devices. Functional integrations are also conducive to monitoring the role of management system play [7].

\section{Wireless Information Transmission Technology}

Wireless information transmission technology refers to the use of electromagnetic waves for information transfer, so that the transmission medium does not require a tangible line to achieve. Relative to the wired information transmission technology, wireless transmission technology is relatively low cost, its own performance is relatively stable, the most critical point is that it is more convenient and quick. Now, with the widespread use of wireless routers, wireless information transmission technology gradually broad application [8].

Information and communication engineering technology from the moment of emergence, is associated with people's lives. From the original wireless transmission signal to the later common household appliances in the remote control, to now more common commercial wifi and home wifi, are infinite information transmission technology applications. Because the wireless information transmission technology has the characteristics of simple operation, plug and play, convenient management and so on, it does not need to use visible lines, so the requirements for the transmission environment is low, making its application spread rapidly. The application of home wifi makes people meet the needs of residential aesthetics, simple, but also to meet people's needs for the network.

Wireless transmission technology can also achieve network alarm and theft prevention with wireless location technology can be easily achieved. At present, with the gradual improvement of wireless transmission technology, China can be seen everywhere alarm system, which uses the most extensive and most mature telephone dialing, mainly because the dial-up operation is the most simple, and its system more stable, the network data relative Is also quite reliable, and thus has been widely loved.

\section{The Development Trend of Transmission Technology in Communication Engineering}

Transmission technology itself has a strong development characteristics of the times, will be highly efficient and scientific one together, the development of information and communication engineering development in the information security is of great significance. Therefore, in the future, we will continue to strengthen the momentum of innovation in transmission technology, and continuously strengthen the specific application of transmission technology in communication engineering. It will promote the development of information and communication engineering and 
improve the quality and efficiency of information service.

As far as the function of transmission technology is concerned, the current and even future development has diversified features. From the overall development of information and communication engineering point of view, we can clearly see the future development of transmission technology functions will tend to diversification.

The diversified characteristics of the transmission technology, the performance of the practical application of various devices in information and communication engineering and the function will be correspondingly improved, can make information and communication engineering network access more stable, signal transmission more justified, which has Which will enhance the effect of transmission technology in practical application and improve the stability of information and communication engineering. At the same time, to adapt to the characteristics of small size, the future of communication equipment can only rely on a small device to achieve the various needs of information and communication, so as to achieve the ideal state of information transmission technology, which is the future of transmission technology development. The Important trends [10]. On the one hand to optimize the overall situation of the communication project on the allocation of resources, so that the relevant staff can be developed for the specific circumstances of the corresponding information and communication programs, on the other hand can also promote the further development of transmission technology to promote information and communication quality of service.

Second, the development of transmission technology is another major trend of commercial development. Information and communication engineering development to the next stage, information transmission technology is bound to be further application development, so in the commercial distribution of the market continue to reduce the cost of transmission technology and promote technological innovation and improvement.

Finally, the transmission technology and N1STP technology is a major trend. Based on the traditional transmission technology, ASON can greatly enhance the bandwidth utilization and achieve the goal of cost reduction. This will enable operators to develop a variety of voice and data services using the backbone layer and large metropolitan area network according to their own needs. In this respect, optical fiber transmission technology and wireless transmission technology has a considerable advantage.

\section{Conclusion}

With the rapid development of Chinese economy and society, the Internet era has come full, and the corresponding, people for the Internet and information and communication engineering information transmission technology requirements are also rising. Information transmission technology as information and communication engineering in the most critical part of the need for people to provide safe, convenient and non-destructive information transmission. The main transmission technology, wired optical fiber transmission technology and wireless transmission technology is the most important means of transmission, optical fiber transmission through its high capacity, high efficiency is known as the "information superhighway", while the wireless transmission technology is to rely on Its more efficient and flexible advantages have been widely loved. Therefore, the only way to improve the information transmission technology and level are to provide people with better information services, thus promoting the information technology.

\section{References}

[1] Xi Wei. Transmission technology in the effective application of information and communication engineering analysis [J] China New Communications, 2015,24: 91.

[2] Sun Hongguang. Application and development trend of transmission technology in communication engineering [J] .Journal of China New Telecommunications, 2016,03: 48-49.

[3] Reza Wanguly Aihaiti. Transmission technology in the effective application of information and 
communication engineering [J]. Information and Communication, 2016, 04: 204-205.

[4] Liu Bin, Du Chuanhui. On the transmission technology in the communication engineering application and development direction [J]. Western leather, 2016, 14: 3.

[5] Shao Shuai. Application of transmission technology in information and communication engineering [J]. Digital Technology and Applications, 2016, 05: $27+29$.

[6] Fang Xiang. Transmission technology in the application of information and communication engineering analysis [J] China New Communications, 2016,17: 120-121.

[7] Chen Jingwei. Transmission technology in the effective application of information and communication engineering analysis [J]. Science and Technology Innovation Herald, 2013,01: 129.

[8] Tan Zhuoting. Transmission technology in the effective application of information and communication engineering [J]. China New Communications, 2013, 14: 41-42.

[9] Sun Xiaojin. Application analysis of transmission technology in information and communication engineering [J]. Journal of Computer Software and Application, 2013, 17: 299-300.

[10] Liang Fei. On the transmission technology in the application of information and communication engineering [J]. Enterprise Technology and Development, 2015, 15: 26-27. 